\title{
Statistical Methods for Providing the Stability of the Solutions of Inverse Problems and Their Application to Decrease Rotor Vibroactivity
}

\author{
A. V. Goroshko and V. P. Roizman \\ Khmelnytskyi National University, Khmelnytskyi, Ukraine \\ e-mail: iftomm@ukr.net; royzman_V@mail.ru \\ Received November 6, 2013
}

\begin{abstract}
Many machine design problems relate to the class of inverse problems, whose solution is reduced to the solution of systems of linear algebraic equations. The relation between the matrix conditionality number of such systems and solution errors is shown in this article. The application of the least square method to improve the accuracy of solutions is justified. A method for providing the stability of solutions of systems of linear algebraic equations based on the use of additional information by increasing the number of measurements of initial parameters is proposed. The approach and statistical algorithm for achieving a given accuracy of the solution of poorly specified systems of linear algebraic equations are developed. The results of using the offered methods for the identification of eccentricities and elastic inertial parameters in the problem of decreasing the vibroactivity of the rotor of turbine pump are given.
\end{abstract}

DOI: $10.3103 / \mathrm{S} 1052618815030073$

Most practical problems, including machine design, optimization of technological processes, and diagnostics of technical state, are inverse. Their solution is reduced to solving systems of linear algebraic equations. However, errors in the initial data should be taken into account due to the incorrectness of the inverse problem.

\section{SOLUTION ERROR ESTIMATION}

Let us consider a linear model of the following form:

$$
\mathbf{y}=\mathbf{A x}=x_{1} \mathbf{a}_{1}+x_{2} \mathbf{a}_{2}+\ldots+x_{m} \mathbf{a}_{m},
$$

where $\mathbf{x} \in \mathbf{R}^{m}$ is the vector of unknown values (object), $\mathbf{y} \in \mathbf{R}^{n}$ is the vector of observation data, $\mathbf{a}_{i} \in \mathbf{R}^{n}$, $i=1,2, \ldots, m$ are the column vectors of matrices $\mathbf{A}, \mathbf{A} \in \mathbf{R}^{n \times m}$. Matrix $\mathbf{A}$ can be considered to be the mathematical expression of the linear operator $\mathbf{A}$, implementing the reflection $\mathbf{R}^{m} \longrightarrow \mathbf{R}^{n}$.

The pseudosolution of system (1) is

$$
\mathbf{x}=\mathbf{A}^{+} \mathbf{y},
$$

where $\mathbf{A}^{+}=\left(\mathbf{A}^{\mathrm{T}} \mathbf{A}\right)^{-1} \mathbf{A}^{\mathrm{T}}$ is the Moore-Penrose pseudoinverse matrix [1]. Specifically, if $m=n$ and $\mathbf{A}$ is the square nonsingular matrix, the solution (2) turns into the normal (inverse) one:

$$
\mathbf{x}=\mathbf{A}^{-1} \mathbf{y} .
$$

However, in spite of the possibility of finding solution (2) in principle and its uniqueness, practice shows that the problem becomes more complicated when the matrix conditionality number is large, and the solution is instable. Considering the case where $m=n$ without loss of generality, let us determine the estimation of the relative error of the required value. Let the following system be solved, instead of (1):

$$
\left(\mathbf{A}_{0}+\Delta \mathbf{A}\right)\left(\mathbf{x}_{0}+\Delta \mathbf{x}\right)=\mathbf{y}_{0}+\Delta \mathbf{y}
$$

i.e., the elements of matrix, required vector, and right part have some unknown absolute errors $\Delta \mathbf{A}, \Delta \mathbf{x}, \Delta \mathbf{y}$ depending on the accuracy of the control equipment and other factors and being small in comparison to the true values of $\mathbf{A}_{0}, \mathbf{x}_{0}, \mathbf{y}_{0}$. Let us denote the relative errors by $\delta_{A}=\|\boldsymbol{\Delta} \mathbf{A}\| /\left\|\mathbf{A}_{0}\right\|, \delta_{x}=\|\boldsymbol{\Delta} \mathbf{x}\| /\left\|\mathbf{x}_{0}\right\|, \delta_{y}=$ $\|\Delta \mathbf{y}\| /\left\|\mathbf{y}_{0}\right\|$. After simple transformations, we obtain 


$$
\delta_{x} \leq \operatorname{cond}\left(\mathbf{A}_{0}\right) \delta_{y}+\left[\operatorname{cond}\left(\mathbf{A}_{0}\right)\right]^{2} \delta_{A}
$$

where cond $(\mathbf{A})=\left\|\mathbf{A}_{0}^{-1}\right\| \cdot\left\|\mathbf{A}_{0}\right\|$ is the conditionality number of matrix $\mathbf{A}$. If the matrix elements are accurately specified, the inequality (5) takes the form

$$
\delta_{x} \leq \operatorname{cond}(\mathbf{A}) \delta_{y} .
$$

Here, the quantitative relationship between the solution accuracy, the selected mathematical model, and equipment accuracy is given. It is seen from (6) that the maximum relative error of the solution is

$$
\left(\delta_{x}\right)_{\max }=\operatorname{cond}\left(\mathbf{A}_{0}\right) \delta_{y} .
$$

This dependence allows one to determine a factor using two factors, namely, to define the equipment for successful measurement for the existing mathematical model and the given accuracy, to estimate the solution accuracy by the equipment and mathematical model, or to determine the efficiency of the selected model by the given method accuracy and the control equipment.

It is known from [2] that matrix conditionality in the Euclidian form is equal to the relationship between the maximum and minimum modules of its eigenvalues

$$
\operatorname{cond}(\mathbf{A})=\left(\max \left|\lambda_{i}\right|\right) /\left(\min \left|\lambda_{i}\right|\right),
$$

i.e., the conditionality number and, then, the solution error probably increases with a growing matrix dimension.

Since it is impossible to define the maximum distance between the approximate and true solutions exactly using (6) because the true values are unknown in advance, more meaningful results can be obtained on calculating the assessments of absolute error of solution. In particular, the absolute error of solution for $m=n$ can be defined as

$$
\sigma_{\min }\|\Delta \mathbf{x}\|_{\max }=\|\Delta \mathbf{y}\|
$$

$\sigma_{\min }>0$ is the minimum singular number in the singular decomposition $\mathbf{A}[1]$.

\section{CONSTRUCTION OF APPROXIMATE SOLUTIONS WITH A GIVEN ACCURACY}

Since it is impossible to find the exact solution at poor conditionality (1) and measurement errors, let us consider $\mathrm{N}$ approximate solution. In this case, deterministic and probabilistic approaches are feasible in dependence of the nature of initial data. Taking into account that experimental data are always random, the formulation of the inverse problems in the context of the statistic estimation theory of unknown parameters is the most natural. As opposed to the numerical characteristics, the estimations are random, and their values depend on the volume of experimental data, while the probability distribution laws relies on the distribution of probability of the random numbers or values of measured magnitudes.

All known solution methods for poorly conditioned systems of linear algebraic equations, which are called regularizing algorithms [3-6], are aimed at increasing the system's stability by a decrease in the matrix conditionality number.

Considering the statistical nature of the measured output characteristics, new approaches to the increase of the solution accuracy of the systems of algebraic equations can be applied. This can be summarized by the following. Since the problem at $m=n$ is instable, the data on the object is assumed to be extended by increasing the number of equations $n \gg m$ to obtain the overdetermined system of equations. In practice, this means a growing number of measurements. Strictly speaking, an overdetermined system is inconsistent, and, therefore, the solution should be found in the form of unified object $\hat{\mathbf{x}}$, whose substitution into the left part of (1) instead of $\mathbf{x}$ provides the most accurate presentation of the sum total of the experimental data [7]. To do this, let us use the maximum-likelihood method. Though the estimation by this method is instable, its region is always narrower than the one of the admissible valuation for (2). Moreover, the maximum-likelihood estimation approaches $\mathbf{x}$ with an increase in the number of implementations $n$. Likelihood maximization requires that discrepancy $\left(\hat{\mathbf{x}}-\mathbf{x}_{0}\right)^{2}$ is minimum. For normal error distribution, the estimation is implemented by the least square method.

Figure 1 shows the results of numerical experiment for the system of linear algebraic equations with cond $(\mathbf{A})=86.99$, wherein the measurement results were modeled at $n=1000$. The center of the region formed from the least square estimations is shifted from $\mathbf{x}_{0}$. In this case, $\hat{\mathbf{x}} \longrightarrow \mathbf{x}_{0}$ with increasing $n$. 


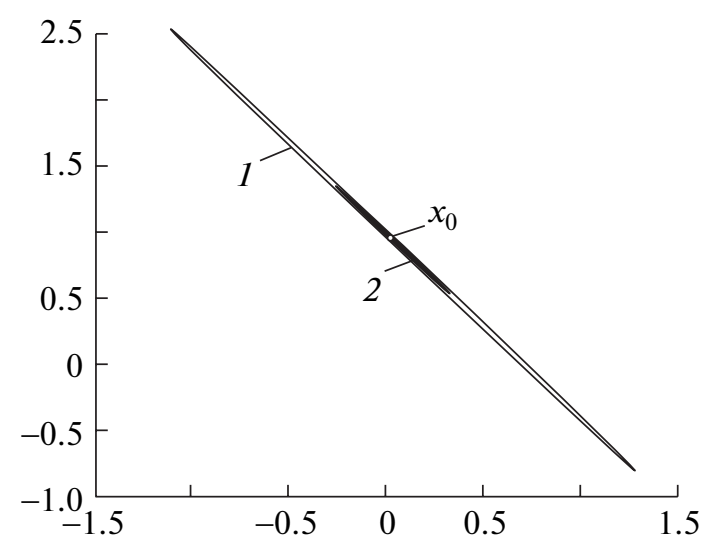

Fig. 1. Illustration of the solutions of linear algebraic equation systems: (1) permissible error region; (2) region formed by the least square method estimations.

Estimations (7), (8), and (9) give only maximum error and do not allow one to judge how close a certain solution is to the correct one. The obtained calculation result depends, among other factors, on specific or unknown error occurring in the measurement instant. At any other time instant, the measurement error of the same parameter from the same device can be different. However, the values of the errors of measured magnitudes have a top limit of the class of instrument accuracy, i.e., the random values with mathematical expectation equal to their true value and the mean square deviation equal to the third part of the instrumental accuracy. Therefore, the elements resulted from solving system (1) over multiple measurements of $\mathbf{Y}$ and $\mathbf{A}$ under the same conditions can attain arbitrary values inside the limited region, whose value is determined by estimations (7) and (9). This means that the random values obeying certain distribution laws are considered. If the mathematical expectation is found for each factor taken as the random value, it will closer to the true results than others.

In practice, there is often a possibility to conduct several measurements $l$ so that $l=k * m$, i.e., to carry out $k \in \mathbf{N}$ measurements for each parameter $y_{1}$. In this case, it is suggested to take $m=n$ instead of finding the estimation by the least square method and calculate the inverse solution (3), substituting the averaged value $\bar{y}_{i}=\sum_{j=1}^{k} y_{i j}, i=1,2, \ldots, n$ for $y_{i}$. The obtained estimation $\hat{\mathbf{x}}$ coincides with the one determined by the least square method. The given method is substantially the statistical regularization method.

Estimation $\hat{\mathbf{x}}$ will allow one move to the method for providing the given accuracy of solution of (1), namely, defining the necessary number of measurements at the given accuracy. In practice, the accuracy will increase with growing $k$ until random error becomes comparable with systematic error, which can be assessed by comparing the solution of the direct problem, resulting from the Monte Carlo method and having the known input values, with the measured values. Taking into account systematic error will enable clarification of the solution of the inverse problem. In general, to increase the accuracy of solution of (1), it is reasonable to combine the offered and known regularization methods.

\section{PROVIDING SPECIFIED ACCURACY OF POORLY CONDITIONED SYSTEMS OF LINEAR ALGEBRAIC EQUATIONS}

It is evident from (7) that cond(A) or $\delta_{y}$ need to be decreased to increase accuracy. Since the methods of improving the conditionality of matrix $\mathbf{A}$ are enough widely described in literature, let us consider the ways of decreasing $\delta_{y}$. The point is to define how many measurements are sufficient to achieve the given accuracy of (1). Let us take the distribution density $y_{i}$ to be normal $y_{i} \sim N\left(M, \sigma^{2}\right), i=1,2, \ldots, n$. Then $\mathbf{y}$ is the multidimensional normal value $\mathbf{y} \sim N(\boldsymbol{\mu}, \boldsymbol{\Sigma})$, where $\boldsymbol{\mu}=\mathbf{M}(\mathbf{y})$ is the vector of mathematical expectation; $\boldsymbol{\Sigma}=\operatorname{cov}(\mathbf{y})$ is the covariance matrix $n \times n$. To decrease $\delta_{y}$ and, therefore, $\|\Delta \mathbf{y}\|$, it is assumed that $k$ measurements are conducted for $\mathbf{y}$ and the sample estimation $\mathbf{M}(\mathbf{y})=\sum_{i=1}^{k} \mathbf{y}_{i}$ is put for $\mathbf{y}$ in (1). In this case, the relative error is $\delta_{y} \approx\left\|\mathbf{M}(\mathbf{y})-\mathbf{y}_{0}\right\| /\left\|\mathbf{y}_{0}\right\|$.

The number of $k$ measurements of value $\mathbf{y}$ required to achieve the specified accuracy $\delta_{x}$, can be set as 1 follows. It is known that $\lim _{k \rightarrow \infty} \mathbf{M}(\mathbf{y})=\mathbf{y}_{0}$. In real problems, the estimation $\mathbf{M}(\mathbf{y})$ ta limited $k$ allows one to determine the mathematical expectation with some error.

Therefore, calculating $\delta_{y}$ can be replaced by finding the relative error of the mathematical expectation estimation. Let us assess it by expressing $\mathbf{M}(\mathbf{y})$, which results from $k$ measurements, using the recurrent formula

$$
\mathbf{M}(\mathbf{y})_{k}=\left[(k-1) \mathbf{M}(\mathbf{y})_{k-1}+\mathbf{y}_{k}\right] k^{-1}
$$

Where $\mathbf{M}(\mathbf{y})_{k}$ is the estimation of the mathematical expectation at the sampling volume $k ; \mathbf{M}(\mathbf{y})_{k-1}$ is the estimation of the mathematical expectation at the sampling volume $k-1$. 
Let us determine the absolute value of the relative error of evaluating mathematical expectation as

$$
\delta_{y}=\left\|\left(\mathbf{M}(\mathbf{y})_{k}-\mathbf{M}(\mathbf{y})_{k-1}\right) \mathbf{y}_{0}^{-1}\right\| .
$$

Taking into account that the maximum value of the error is $\left\|\mathbf{M}(\mathbf{y})_{k-1}\left(k \mathbf{y}_{0}\right)^{-1}\right\|$, we have the following, after the transformations of (10) and (11): $\delta_{y} \leq\left\|\mathbf{M}(\mathbf{y})_{k-1}\left(k \mathbf{y}_{0}\right)^{-1}\right\|$.

Since $\lim _{k \rightarrow \infty} \mathbf{M}(\mathbf{y})_{k-1}=\mathbf{y}_{0}$ can be taken for large $k$. Hence, to provide the given accuracy $\delta_{y} \leq 1 / k$ of the measurements of vector $\delta_{y}$, the number of measurements should be $k \geq 1 / \delta_{y}$, while

$$
k \geq \operatorname{cond}(\mathbf{A}) / \delta_{x} .
$$

to provide the given accuracy $\delta_{x}$ of solution of (1).

Inequality (12) does not require prior information about $\mathbf{x}_{0}$. There is a need to know the conditionality number of matrix A and the desired value of the relative error of the result. Relationship (12) can be applied to provide the specified accuracy of solution for relatively small cond(A), but it is difficult to do for large conditionality numbers. For instance, the required number of measurements is $k=20000$ at $5 \%$ accuracy and cond $(\mathbf{A})=1000$, which is practically impossible to be implemented. In this case, the approximate statistical algorithm for calculating the necessary number of measurements at the given accuracy of solution of the systems of algebraic equations is suggested. The algorithm is based on the estimation of the number of measurements $k$ that is required to obtain $\mathbf{M}(\mathbf{y})$ with the specified error and confidential probability, which lead to smaller values of $k$. For normal distribution density $y_{i} \sim N\left(M, \sigma^{2}\right), i=1,2, \ldots, n, \mathbf{y}$ is a multidimensional normal value with distribution density

$$
f(\mathbf{y})=\left(\sqrt{(2 \pi)^{n}|\mathbf{\Sigma}|}\right)^{-1} \exp \left[-\frac{1}{2}(\mathbf{y}-\boldsymbol{\mu}) \mathbf{C}^{-1}(\mathbf{y}-\boldsymbol{\mu})\right], \quad \mathbf{y} \in \mathbf{R}^{n},
$$

where $\boldsymbol{\mu}=\mathbf{M}(\mathbf{y})$ is the vector of mathematical expectation $\mathbf{y}, \boldsymbol{\Sigma}=\operatorname{cov}(\mathbf{y})$ is the nonnegative definite symmetric square covariance matrix $n \times n$, which diagonal contains the dispersions of the vector components, while the external diagonal elements are the covariances between the components $\mathbf{y}_{i} ;|\boldsymbol{\Sigma}|=\operatorname{det}(\boldsymbol{\Sigma})$ is the determinant of matrix $\boldsymbol{\Sigma}$, and $\boldsymbol{\Sigma}^{-1}$ is the inverse matrix.

For the number of measurements $k$, the sampling covariance matrix is

$$
\boldsymbol{\Sigma}=(k-1)^{-1} \sum_{i=1}^{k}\left(\mathbf{y}_{i}-\overline{\mathbf{y}}\right)\left(\mathbf{y}_{i}-\overline{\mathbf{y}}\right)^{T}, \quad \text { where } \quad \overline{\mathbf{y}}=k^{-1} \sum_{i=1}^{k} \mathbf{y}_{i} .
$$

The basic method for estimating the parameters of multidimensional random values is the method of sampling analysis. Its concept is to analyze the sampling and spreading the results on the general total sum of the investigated random vector. Let us assess the region for $\boldsymbol{\mu}$. Let the vector of average sampling $\overline{\mathbf{y}}$ and the covariance estimation matrix $\boldsymbol{\Sigma}$ be known. For $n>1$, the Hotelling statistics $T^{2}$ is used in constructing the confidence region. It is concerned with the Fisher $F$-statistics for a given confidential probability $P$ according to the following relationship:

$$
T^{2}=[n(k-1) /(k-n)] F .
$$

where $k$ is sampling volume. Taking (14) into account, the confidence region for $\mu$ with the probability $P$ is described by the following surface equation

$$
(\overline{\mathbf{y}}-\boldsymbol{\mu}) \boldsymbol{\Sigma}^{-1}(\overline{\mathbf{y}}-\boldsymbol{\mu})=[n(k-1) /(k(k-n))] F_{1-P},
$$

where $F_{1-P}$ is the value $F$ at the significance level $\alpha=1-P$ and numbers of freedom degrees $f_{1}=n$ and $f_{2}=k-n$. The confidence region in $\mathbf{R}^{n}$ is an $n$-dimensional ellipsoid with the center in $\overline{\mathbf{y}}$ since $(\overline{\mathbf{y}}-\boldsymbol{\mu}) \boldsymbol{\Sigma}^{-1}(\overline{\mathbf{y}}-\boldsymbol{\mu})$ is the positive definite square form.

The relationship (15) is suitable for determining the permissible error $\delta_{y}=\|\overline{\mathbf{y}}-\boldsymbol{\mu}\| /\|\boldsymbol{\mu}\|$ under the presence of results of $k$ measurements. Assuming that $\lim _{k \rightarrow \infty} \mathbf{M}(\Delta \mathbf{y})=0, \boldsymbol{\mu}=\mathbf{y}_{0}$, (15) is transformed as follows:

$$
(\boldsymbol{\Delta y})^{T} \boldsymbol{\Sigma}^{-1} \Delta \mathbf{y}=[n(k-1) /(k(k-n))] F_{1-P} .
$$




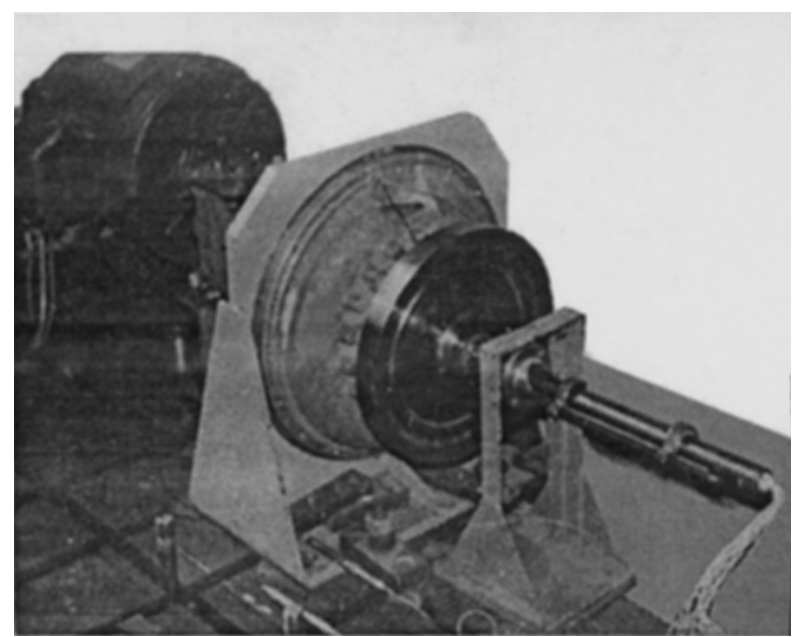

Fig. 2. Layout of the rotor of turbine pump TNA-150.

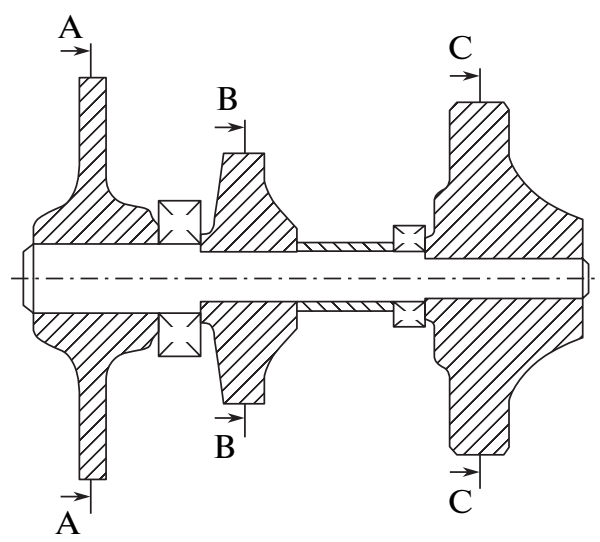

Fig. 3. Design diagram of turbine pump TNA-150.
The required number of measurements $k$ can be approximately found from (16) to achieve the given accuracy of solution of (1). To do this, an inertial algorithm is proposed. This algorithm is used for the given confidential probability $P$ and the permissible solution error $\left\|\Delta \mathbf{x}_{\text {perm }}\right\|$. Before starting the algorithm, $k$ measurements of vector $\mathbf{y} \in \mathbf{R}^{n}$ are implemented. The operation of the algorithms can be presented as three repeating steps.

Step 1 (the error calculation). Sampling average $\overline{\mathbf{y}}^{(t)}$, the inverse covariance matrix $\left(\boldsymbol{\Sigma}^{-1}\right)^{(t)}$, and the value of Fisher $F$-statistics $\left(F_{1-P}\right)^{(t)}$ are calculated. Using (17), $\boldsymbol{\mu}^{(t)}$ and the measurement error $\Delta \mathbf{y}^{(t)}=$ $\overline{\mathbf{y}}^{(t)}-\boldsymbol{\mu}^{(t)}$ are determined. And the value of maximum error of solution $\|\boldsymbol{\Delta} \mathbf{x}\|^{(t)}$ is found from (9).

Step 2 (comparison). The maximum error of solution $\|\mathbf{\Delta} \mathbf{x}\|^{(t)}$ is compared with permissible error $\left\|\Delta \mathbf{x}_{\text {perm }}\right\|$. If $\|\boldsymbol{\Delta} \mathbf{x}\|^{(t)} \leq\left\|\boldsymbol{\Delta} \mathbf{x}_{\text {perm }}\right\|$, the satisfactory accuracy is obtained; if $\|\Delta \mathbf{\Delta x}\|\left\|^{(t)}>\right\| \Delta \mathbf{x}_{\text {perm }} \|$, the coefficient of the error increase $\xi=\|\mathbf{\Delta x}\|^{(t)} /\left\|\boldsymbol{\Delta} \mathbf{x}_{\text {perm }}\right\|$ is calculated.

Step 3 (correction and clarification). The "corrected" vector of errors $\Delta \mathbf{y}^{(t+1)}=\Delta \mathbf{y}^{(t)} / \xi$ is calculated. Then the value of $k^{(t+1)}$ is determined from (16). Since $k^{(t+1)}$ was estimated by using the results of the first step, it needs to be clarified. Thus, the Monte Carlo method is used to generate the $k^{(t+1)}$ vectors of experimental data with the measurements errors being equal to the conducted $k$ tests.

The inertial process terminates when $\|\boldsymbol{\Delta} \mathbf{x}\|^{(t)} \leq$ $\left\|\boldsymbol{\Delta} \mathbf{x}_{\text {perm }}\right\|$. The algorithm convergence is achieved by the assured existence of the solution of Eq. (16). The algorithm can be implemented on the basis of known optimization methods and such software as Matlab.

\section{DETERMINING THE ECCENTRICITY \\ IN THE TURBINE PUMP ROTOR BALANCING PROBLEM}

The presented method for solving the inverse problems was applied to increase the vibroactivity of the turbine pump TNA-150. For this purpose, rotor eccentricity, as well as inertial parameters with a further calculation of critical values, was defined. Turbine pumps containing the fast-turning rotors are widely used in rocket, aviation, chemical, and other industries. The examined type of the unit had the increased vibrations induced by the rotor misbalance. This is why there was a need to decrease the rotor deformations, stresses, and load on its bearings to $300 \mathrm{~N}$ by compensating for operational eccentricities and misbalances of largest parts mounted on the shaft.

The shaft of the turbine rotor on two bearings contains the impellers of two compressor stages and a disc of axial turbine (Fig. 2). Since the main masses and misbalances are concentrated exactly here, a three-mass design diagram for the rotor was selected (Fig. 3). For the given mathematical model of rotor oscillations to be effective, the elastic inertial characteristics of the rotor were identified by measuring the bends on four rotational frequencies $\omega_{j}, j=1,2,3,4$.

To find the rotor motion equations, bending theory equations [8] were used. As a result, equations relating unknown distributions of rigidity $E J$, masses $m$, and eccentricity $e$ with the rotor bends $y$ were 
written in projections for two mutually perpendicular planes for each of three cross-sections of the rotor (Fig. 3).

$$
\alpha_{o} K_{z z}^{\prime \prime}\left(Z, \omega_{j}\right)+2 \alpha_{1} K^{\prime}\left(Z, \omega_{j}\right)+\alpha_{2} K\left(Z, \omega_{j}\right)-e_{y} \omega^{2}=\omega_{j}^{2} y
$$

where $\alpha_{i}=\alpha_{i}(Z)=\frac{1}{m} \cdot \frac{d^{(i)} E J}{d Z^{i}}, i=0,1,2$ are the statistical influence coefficients; $K(Z, \omega)=y^{\prime \prime} /\left[1+\left(y^{\prime}\right)^{2}\right]^{3 / 2}$ is the curvature of the elastic line of the rotor; and $Z$ is the coordinate of the rotor cross-section counted along the rotation axis. The bends were measured in the sections $\mathrm{A}, \mathrm{B}$, and $\mathrm{C}$ in the whole range of rotational frequencies $0-18000 \mathrm{rpm}$, and their values were selected at 14100, 15000, 15600, and $16000 \mathrm{rpm}$. The projections of these bends and their derivatives were put into two systems of equations (17) for each section, obtaining systems of four independent equations.

To solve the systems (17), their conditionality numbers were calculated and proved to be in the range of 3.2-6.7. Therefore, the possible error of determining the unknown values can be up to $134 \%$ for an instrumental accuracy of $15 \%$ for measuring the bends. To improve calculation accuracy, the developed statistical method for increasing the stability of mathematical models was applied. In this case, the bends were measured minimum 50 times, and the mathematical expectations of the values and phases of these bends were found. These average values were put into (17). Using the resulting eccentricities $e_{x}$ and $e_{y}$ and the influence coefficients $\alpha_{0}$ and $\alpha_{1}$ for each cross section, the values of reduced masses and rigidities of the shaft as well as the shaft portions with the disks $M_{i}, i=1,2,3$ were found by the following formulas:

$$
m(Z)=M \exp \left(\int_{0}^{Z}\left(\alpha_{0}\right)^{-1} \alpha_{1} d Z\right), \quad E J(Z)=m(Z) \alpha_{0}(Z)
$$

Where $M$ is the mass of the rotor.

The results of the solution:

For the first section: $e_{x}=-5 \times 10^{-6} \mathrm{~m} ; e_{y}=-5.84 \times 10^{-6} \mathrm{~m} ; E J_{1}=414.7 \mathrm{~N} / \mathrm{m}^{2} ; \quad m_{1}=0.22 \times 10^{-2} \mathrm{~kg} / \mathrm{m}$; $M_{1}=4.05 \mathrm{~kg}$. For the second section: $e_{x}=-9 \times 10^{-6} \mathrm{~m} ; e_{y}=1.7 \times 10^{-6} \mathrm{~m} ; E J_{2}=1594 \mathrm{~N} / \mathrm{m}^{2} ; m_{2}=$ $0.2 \times 10^{-2} \mathrm{~kg} / \mathrm{m} ; M_{2}=2.74 \mathrm{~kg}$. For the third section: $e_{x}=-6.2 \times 10^{-6} \mathrm{~m} ; e_{y}=30 \times 10^{-6} \mathrm{~m} ; E J_{3}=$ $23998 \mathrm{~N} / \mathrm{m}^{2} ; m_{3}=0.83 \times 10^{-2} \mathrm{~kg} / \mathrm{m} ; M_{3}=11 \mathrm{~kg}$.

The first and second critical frequencies of oscillations calculated by the obtained values $E J, M$, and $m$ appeared to be $n_{1}=16100 \mathrm{rpm}$ and $n_{2}=25080 \mathrm{rpm}$; calculation error did not exceed $3 \%$ of the experimental values. As a result of the implemented high-frequency balancing with the compensation of the eccentricities determined by means of the balancing masses, the bends of the rotor in the rotation frequency range 2000-18000 rpm, the amplitudes of the support vibrations, reactions, statistical stresses in the shaft material, and the dynamic ones increased $6,4,4.5,3.5$, and 3 times, respectively.

\section{CONCLUSIONS}

The application of the least square method to increase the stability of models specified by the systems of linear algebraic equations is proven in the present article. A method for providing the stability of their solution based on an increase in the number of measurements of initial parameters as well as an approach and statistical algorithms to ensure the given accuracy of solving poorly conditioned systems of linear algebraic equations are suggested.

The results of using the methods for identification of the eccentricities, rigidities, and masses in the problem on decreasing the vibroactivity of the turbine pump rotor are presented.

\section{REFERENCES}

1. Andrushevskii, N.M., Analiz ustoichivosti reshenii sistem lineinykh algebraicheskikh uravnenii. Uchebnoe posobie (Solutions Stability Analysis of Linear Algebraic Equation Sets. Student's Book), Moscow: The faculty of Computational Mathematics and Cybernetics of Lomonosov Moscow State Univ.; MAKS Press, 2008.

2. Bellman, R., The stability of solutions of linear differential equations, Duke Math. J., 1943, vol. 10, no. 4, pp. 643-647.

3. Tikhonov, A.N. and Arsenin, V.Ya., Metody resheniya nekorrektnykh zadach (The Way to Solve Incorrect Problems), Moscow: Nauka, 1979. 
4. Ivanov, B.K., Vasin, B.B., and Tanana, B.P., Teoriya lineinykh nekorrektnykh zadach i ee prilozheniya (The Theory of Linear Incorrect Problems and Its Applications), Moscow: Nauka, 1978.

5. Morozov, V.A., Metody regulyarizatsii neustoichivykh zadach (Regularization Procedure of Instable Problems), Moscow: MSU, 1987.

6. Bakushinskii, A.V. and Goncharskii, A.V., Iterativnye metody resheniya nekorrektnykh zadach (Iterative Methods for Solving Incorrect Problems), Moscow: Nauka, 1989.

7. Terebizh, V.Yu., Vvedenie v statisticheskuyu teoriyu obratnykh zadach (Introduction into Statistical Theory of Inverse Problems), Moscow: Fizmatlit, 2005.

8. Osnovy balansirovochnoi tekhniki (Foundations of Balancing Engineering), Shchepetil'nikov, V.A., Ed., Moscow: Mashinostroenie, 1975, vol. 2, pp. 179-252.

Translated by A. Evseeva

SPELL: 1 . ta 